\title{
Contrast variation in SANS with polarized neutrons and polarized samples
}

\section{SCHWAHN}

Forschungszentrum Jülich GmbH, Institut für Festkörperforschung, Postfach 19 13, 52425 Jülich, Germany

\begin{abstract}
An overview of contrast variation in SANS with polarized neutrons and targets is given. Examples from metal physics and biology demonstrate the advantages and the gain of information by this technique.
\end{abstract}

\section{Introduction}

SANS experiments with polarized neutrons provide more detailed information about magnetic properties. Classical SANS experiments in this field are studies of flux lines in superconductors [1] and dislocations in ferromagnetic metals using the magneto elastic effect [6-9]. The study of flux lines in high $\mathrm{T}_{\mathrm{c}}$ superconductors is an active field in present research [2-5]. Also investigations of spin wave dynamics in ferromagnets are subject of recent experiments [10]. At present only very few SANS instruments are equipped with the experimental tools to use polarized neutrons, i.e. polarizer, spin flipper and magnetic guide field [11]. Spin analysis of the scattered neutrons has not yet been applied in SANS but seems to be possible in future [11].

In this paper the use of polarized neutrons and polarized targets for measurements with different scattering contrasts will be discussed more systematically. Examples of contrast variation by magnetic scattering and by nuclear spin polarization will be presented. Several principle advantages can be achieved by this technique: (1) One sample is needed instead of several ones as it is the case in isotope contrast variation. (2) The contrast can be strongly increased especially for hydrogen by nuclear spin polarization. (3) Small scattering effects can be extracted from high intrinsic background scattering as incoherent scattering. On the other hand sometimes a large experimental effort and excessive beamtime is necessary.

\section{Basic Theory: Scattering Length Operator}

The scattering length in its general form is defined as a quantum mechanical operator [12-15].

$$
\hat{\mathrm{b}}=\mathrm{b}+\underline{\hat{\sigma}}(\mathrm{b} \mathrm{N} \underline{\hat{I}}+\mathrm{p} \underline{\hat{q}})
$$

$\mathrm{b}, \mathrm{b}_{\mathrm{N}}$ and $\mathrm{p}$ are the coherent scattering lengths for nuclear spin independent, nuclear spin dependent, and magnetic scattering, respectively. Their numerical values can be found in the literature [12]. The vector operators $\underline{\hat{\sigma}}, \underline{\hat{I}}$, and $\underline{\hat{q}}$ are the respective Pauli spin operator for the neutron, the nuclear spin operator, and the operator for magnetic interaction. The magnetic interaction is a long range dipole interaction and therefore the magnetic scattering is anisotropic with respect to the directions of the magnetization $\underline{M}$ of the sample and the scattering vector $\underline{Q}$. 


\section{Magnetic Contrast Variation in Binary Alloys}

The macroscopic cross-section of a two phase structure in an alloy is given by

$$
\frac{\mathrm{d} \Sigma}{\mathrm{d} \Omega}=\phi \mathrm{V}_{\mathrm{p}} \Delta \rho^{2}|\mathrm{~F}(\mathrm{Q})|^{2}
$$

where $\Delta \rho$ is the difference of the coherent scattering length densities of both phases; $\phi, \mathrm{V}_{\mathrm{p}}$, and $\left[\left.\mathrm{F}(\mathrm{Q})\right|^{2}\right.$ are the volume fraction, volume, and form factor of the precipitates, respectively. Correlations between precipitates as the minor phase are neglected in eq. (2). The coherent scattering length density of one phase is given by $\rho=\bar{b} / \bar{\Omega} . \bar{b}$ and $\bar{\Omega}$ are the respective averages of coherent scattering lengths and the atomic volumes.

$A$ two phase structure with the phases $A$ and $B$ and one atom in phase $A$ with the magnetic scattering length $\mathrm{p}$ will be discussed. For such a model we evaluate the contrast from eq. (1)

$$
K=\Delta \rho^{2} \Omega_{A}^{2}=\left[b^{2}+2 c b p \underline{q} \underline{p}_{n}+c^{2} p^{2} q^{2}\right]
$$

with $\mathrm{b}=\left(\mathrm{b}_{\mathrm{A}}-\omega \bar{b}_{\mathrm{B}}\right), \omega=\Omega_{\mathrm{A}} / \Omega_{\mathrm{B}}$. $\mathrm{c}$ is the concentration of the atom with the magnetic scattering length $\mathrm{p}$ in phase $\mathrm{A}$. $\underline{\mathrm{q}}$ is the magnetic interaction vector

$$
\underline{\mathrm{q}}=\underline{\eta}-\underline{\kappa}(\underline{\kappa} \cdot \underline{\eta})
$$

$\underline{\eta}$ and $\underline{\kappa}$ are the respective unit vectors in direction of the magnetization of the sample and of the scattering vector. $\underline{p}_{n}$ is the polarization vector of the neutron.

The first and third term of $\mathrm{K}$ in eq. (3) are independent of the degree of neutron polarization. They describe nuclear and magnetic scattering. In the derivation of eq. (3) from eq. (1) it is assumed that there exists no correlations between the relative orientations of the spins at different positions (page 431 in [15]). Correlations of spin directions between different atoms play a role e.g. in spin waves; in this case an additional term (i $\mathrm{p}^{2} \underline{\underline{\sigma}}\left(\hat{\mathrm{q}}_{\mathrm{i}} \times \underline{\mathrm{q}}_{\mathrm{j}}\right)$ ) appears in eq. (3) with $i, j$ as atomic positions. This term provides the basis of the studies in [10]. The second term in eq. (3) describes the interference between nuclear and magnetic scattering. Next, different possibilities for the contrast are discussed. The sample is assumed to be completely polarized in a saturated magnetic field. If the neutrons are unpolarized with $\left|\underline{p}_{n}\right|=0$ one measures the incoherent superposition of nuclear and magnetic scattering according to

$$
K=\left[b^{2}+c^{2} p^{2} q^{2}\right]
$$

The square of the magnetic interaction vector as evaluated from eq. (4) is $\mathrm{q}^{2}=\sin ^{2} \alpha$ with $\alpha$ as the angle between $\underline{Q}$ and $\underline{\mathrm{M}}$. Thus, from measurements at $\underline{\mathrm{Q}} \| \underline{\mathrm{M}}(\alpha=0)$ and $\underline{\mathrm{Q}} \perp \underline{\mathrm{M}}(\alpha=\pi / 2)$ nuclear and magnetic scattering can be obtained separately.

With polarized neutrons at $\underline{Q} \| \underline{M}$ one measures only nuclear scattering whereas for $\underline{Q} \perp \underline{M}$ the absolute value of $\mathrm{g}$ is unity and directed parallel to $\underline{M}$. Then one measures constructive and destructive interference of nuclear and magnetic scattering for the respective polarization of the neutrons parallel and antiparallel to the magnetization of the sample, namely

$$
\mathrm{K} \pm=\left[\mathrm{b}^{2} \pm 2 \mathrm{cb} \mathrm{p}+\mathrm{c}^{2} \mathrm{p}^{2}\right]
$$

\subsection{Example: Experiments with high intrinsic background}

In one performs two measurements with the same sample and with neutron spin parallel and antiparallel to the direction of the applied magnetic field and then subtracts both spectra one 
gets

$$
\frac{\mathrm{d} \Sigma}{\mathrm{d} \Omega}(\mathrm{Q})=\phi \mathrm{V}_{\mathrm{p}}|\mathrm{F}(\mathrm{Q})|^{2}\left[4 \mathrm{c} \mathrm{b} \mathrm{p} / \Omega_{\mathrm{A}}{ }^{2}\right]
$$

with the contrast $\left(\mathrm{K}_{+}-\mathrm{K}_{-}\right)$from eq. (6). This method is a reliable one to extract small scattering effects from a high background level. Ebel et al. [16] have demonstrated this method in a study of nucleation of Co precipitates in a $\mathrm{Cu}-0.8$ at $\% \mathrm{Co}$ alloy. In this experiment $\mathrm{d} \Omega / \mathrm{d} \Omega$ of eq. (7) could be extracted from an intrinsic background level about ten times higher, namely from incoherent scattering at large $Q$ and the $Q^{-4}$ power law from grain boundaries at small $Q$. However, this intrinsic large background can be avoided by using the isotope $\mathrm{Cu}^{63}$ and a single crystal with polished surfaces [7]. In this way the experiment could be strongly improved and it should also be possible to extract the magnetic scattering. Then one could measure the nuclei of Co precipitates with two different contrasts. This could be important for a more detailed and consistent interpretation of the data.

\subsection{Example: Determination of the gas density for helium bubbles in metals}

A few appm He in metals lead to detrimental changes of the mechanical properties. They are caused by nucleation and growth of bubbles at elevated temperatures. A key parameter for the formation of bubbles is the gas pressure of He in the bubbles and related to the gas density. The gas density can be obtained in a scattering experiment from the contrast in eq. (3)

$$
\mathrm{K}=\mathrm{b}^{2}=\left[\mathrm{b}_{\mathrm{m}}-\omega \mathrm{b}_{\mathrm{He}}\right]^{2}
$$

and evaluated from $\omega=\Omega_{\mathrm{m}} / \Omega_{\mathrm{He}}$ with the known values for $\mathrm{b}_{\mathrm{He}}$, $\mathrm{b}_{\mathrm{m}}$ and $\Omega_{\mathrm{m}}$. The parameters $b_{\mathrm{m}}$ and $\Omega_{\mathrm{m}}$ are the coherent scattering length and atomic volume of the metal atom, respectively. In order to eliminate the unknown form factor in eq. (2) one has to perform two measurements with the same microstructure but with different contrasts. The ratio of both spectra yields $\alpha(\omega)=K_{1} / K_{2}$ from which $\Omega_{\text {He }}$ can be evaluated. Such SANS experiments have been performed using two identically prepared samples with different isotopes, namely a combination of $\mathrm{Ni}^{58}$ and $\mathrm{Ni}^{60}[17]$ and two $\mathrm{Ni}^{58}$ samples implanted with $\mathrm{He}^{3}$ and $\mathrm{He}^{4}$ [18]. In Fig. 1 the experimentally determined function $\alpha(\omega)$ from ref. [18] is shown from two samples after $2 \mathrm{~h}$ annealing at $923 \mathrm{~K}$. $\alpha$ depends on $Q$, its value is around 1 at small $Q$ due to the larger bubbles at the grain boundaries and the sample surface. It is about 0.6 for the smaller bubbles in the bulk. The distribution of the bubbles in the sample was observed by TEM [19]. In Fig. 2 the theoretical $\alpha$ value is plotted as a function of He density $\omega$ for various isotope combinations. Curve 1 has to be used for the analysis of $\alpha$ in Fig. 1. It yields a He gas density of about 0.86 of the atomic density of $\mathrm{Ni}$ for the bubbles in the bulk.

The application of magnetic contrast variation with polarized and unpolarized neutrons can greatly improve the experimental possibilities for the study of He bubbles: Because of the need of only one sample the elimination of the form factor is more reliable and in addition there are several independent combinations of contrast. We discuss $\mathrm{He}^{4}$ in $\mathrm{Fe}$. $\mathrm{Fe}$ is a better candidate than $\mathrm{Ni}$ arising from its nearly 4 times larger magnetic scattering length [12]. For a polarized sample in a magnetic saturation field and unpolarized neutrons one finds nuclear and magnetic scattering, namely $\mathrm{K}(\underline{\mathrm{Q}} \| \underline{\mathrm{M}})=\left[\mathrm{b}_{\mathrm{Fe}}-\omega \mathrm{b}_{\mathrm{He}}\right]^{2}$ and $(\mathrm{K}(\underline{\mathrm{Q}} \perp \underline{\mathrm{M}})-\mathrm{K}(\underline{\mathrm{Q}} \| \underline{\mathrm{M}}))=\mathrm{p}_{\mathrm{Fe}}{ }^{2}$ as derived from eq. (5) and plotted in Fig. 3. Of course the magnetic scattering is independent on He gas density. If one applies polarized neutrons, at $\underline{Q} \| \underline{M}$ nuclear scattering and at $\underline{Q}+\underline{M}$, $\mathrm{K} \pm=\left(\mathrm{b}_{\mathrm{Fe}}-\omega \mathrm{b}_{\mathrm{He}} \pm \mathrm{p}_{\mathrm{Fe}}\right)^{2}$ is obtained as plotted in Fig. 3. $\mathrm{K}_{+}$and $\mathrm{K}$ - are the respective contrast for neutron spin parallel and antiparallel to the magnetization. At $\omega=0$ one gets the contrast of $\mathrm{Fe}$ against vacuum. The magnetic contrast is about half of the nuclear contrast while the contrast with neutron spin parallel to the magnetization $K_{+}$is about 3 times larger than the nuclear contrast. The contrast $\mathrm{K}$. with neutron spin antiparallel to $\underline{\mathrm{M}}$ is small according to the

destructive interference of nuclear and magnetic scattering. In Fig. 4 three combinations of contrasts are plotted. The combination of magnetic and nuclear scattering can be measured with unpolarized neutrons while the other ones can only be obtained with polarized neutrons. A further possible combination of nuclear contrast and $\mathrm{K}$. has not been plotted because of too weak contrast. From Fig. 4 one clearly recognizes that the various combinations of contrasts are 


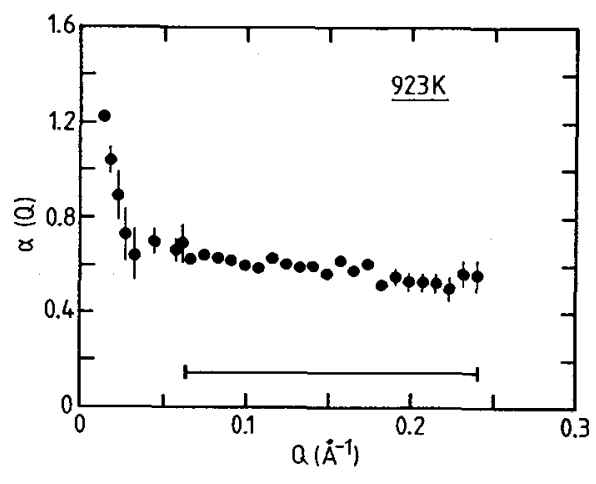

Fig. 1

Experimental $\alpha$ of $\mathrm{He}^{3} / \mathrm{He}^{4}$ in $\mathrm{Ni}^{58}$.

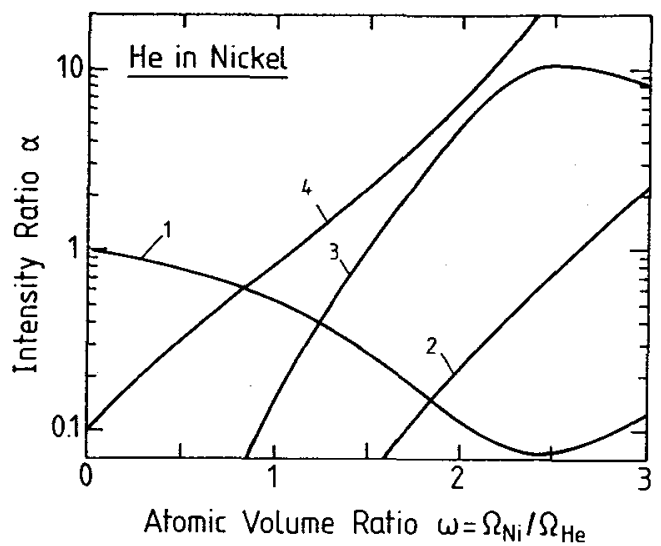

Fig. 2

Calculated $\alpha$ values of $\mathrm{He}$ in $\mathrm{Ni}$ for various isotope combinations:

1.) $\mathrm{He}^{3} / \mathrm{He}^{4}$ in $\mathrm{Ni}^{58}, 2$.) $\mathrm{He}^{4}$ in $\mathrm{Ni}^{60} / \mathrm{Ni}^{58}$,

3. $\mathrm{He}^{3}$ in $\mathrm{Ni}^{60} / \mathrm{Ni}^{58}, 4$.) $\mathrm{He}^{4}$ in $\mathrm{Ni} /\left(\mathrm{Ni}^{62}, \mathrm{Ni}\right)$.

The sample $\left(\mathrm{Ni}^{62}, \mathrm{Ni}\right)$ is an alloy of $72 \% \mathrm{Ni} 62$ and $28 \%$ natural $\mathrm{Ni}$.

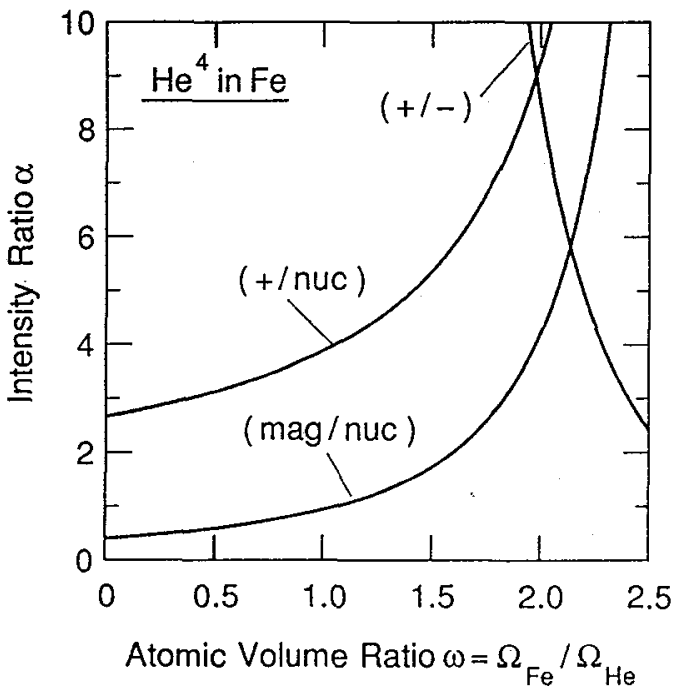

Fig. 3

Various contrasts as a function of He gas density.
Fig. 4

Calculated $\alpha$ of $\mathrm{He}$ in $\mathrm{Fe}$. 
remarkably different as concerns their sensitivity with respect to the He gas density. This clearly demonstrates in addition to the necessity of only one sample the advantages of contrast variation with polarized neutrons.

\section{Contrast Variation by Nuclear Spin Polarization in Biological Molecules}

In this paragraph we present a short discussion of nuclear spin polarization method applied to the ribosome of E-coli, drawn schematically in Fig. 5. Contrast variation is absolutely necessary to perform structural investigations of the complicated shape and inner structure of the ribosome. The cross-section for a molecule with an inner structure as in Fig. 5, dissolved in a solvent with the coherent scattering length density $\rho_{\mathrm{e}}$, is proportional to (eq. (2))

$$
\frac{\mathrm{d} \Sigma}{\mathrm{d} \Omega}(\mathrm{Q}) \propto\left|(\Delta \rho \mathrm{F})_{\mathrm{p}}+(\Delta \rho \mathrm{F})_{\mathrm{A}}+(\Delta \rho \mathrm{F})_{\mathrm{PT}}+(\Delta \rho \mathrm{F})_{\mathrm{M}}\right|^{2}
$$

which is the square of the sum of the scattering amplitudes from the different parts of the molecule with $\Delta \rho_{\mathrm{i}}=\rho_{\mathrm{i}}-\rho_{\mathrm{e}}$. A very attractive possibility of contrast variation is the nuclear spin polarization of hydrogen or deuterium in the molecules whose contrast

$$
\mathrm{K}=\left[\mathrm{b}^{2}+2 \mathrm{~b} \mathrm{~b}_{\mathrm{N}} \mathrm{I} \underline{\mathrm{p}}_{\mathrm{n}} \underline{\mathrm{p}}_{\mathrm{N}}+\mathrm{b}_{\mathrm{N}}^{2} \mathrm{I}^{2} \mathrm{p}_{\mathrm{N}}^{2}\right]
$$

has the same form as the magnetic contrast in eq. (3). $\underline{p}_{\mathrm{N}}$ is the polarization vector of the nucleus and $I$ is its spin quantum number. The techniques of nuclear spin polarization are described in [15] and [20]. In Fig. 6 the contrast of a hydrogen and deuterium target in vacuum are plotted as a function of the degree of nuclear spin polarization for polarized and unpolarized neutrons parallel and antiparallel to the nuclear spin polarization. At $\left|p_{N}\right|=0$ the contrasts of $\mathrm{H}$ and D shows a very small difference which is the basis for most SANS experiments in polymer research. However, if one polarizes the nuclear spin a strong increase of the hydrogen contrast of nearly two orders of magnitude is observed and three different values of the contrasts are accessible depending on the nuclear spin orientation with respect to that of the polarization of the neutrons. A smaller change of contrast is observed for deuterium.

If one likes to position the subunit $A$ in the E-coli ribosome in Fig. 5 one has to protonate subunit $A$ and deuterate the rest of the molecule. From eq. (1) and (9) one then gets

$$
\frac{\mathrm{d} \Sigma}{\mathrm{d} \Omega}(\mathrm{Q}) \propto\left|\mathrm{U}(\mathrm{Q})+\mathrm{c} \mathrm{b}_{\mathrm{N}}^{\mathrm{H}} \hat{\underline{\sigma}} \hat{\underline{L}} \mathrm{~F}_{\mathrm{A}}(\mathrm{Q})\right|^{2}
$$

with $\mathrm{U}(\mathrm{Q})=\Sigma\left(\Delta \rho^{0} \mathrm{~F}(\mathrm{Q})\right)_{\mathrm{k}}$, the spin independent scattering amplitude of the whole molecule. It is assumed that only the hydrogen nuclear spins are polarized. As in magnetic contrast variation one finds three basic scattering functions:

(1) Spin independent scattering $|U(Q)|^{2}$ for $\left|\underline{p}_{N}\right|=0$

(2) an incoherent superposition of spin independent and spin dependent scattering $\left[|\mathrm{U}(\mathrm{Q})|^{2}+\mathrm{c}^{2} \mathrm{~b}_{\mathrm{N}}^{\mathrm{H}} \mathrm{I}^{2}\left|\mathrm{~F}_{\mathrm{A}}\right|^{2}\right]$ with unpolarized neutrons, and

(3) a coherent superposition of both scattering contributions for polarized neutrons with the interference term $\operatorname{Re}\left\{\mathrm{U} \mathrm{F}_{\mathrm{A}}{ }^{*}\right\}$. From $\left|\mathrm{F}_{\mathrm{A}}\right|^{2}$ the shape and size of the subunit is obtained from the interference term $\operatorname{Re}\left\{\mathrm{UF}_{\mathrm{A}}{ }^{*}\right\}$ the position of the subunit in the molecule in principle can be evaluated.

The situation described so far is ideal because a complete protonation and deuteration of subunits is not possible. Therefore, the scattering from a subunit cannot be extracted from the total scattering without contributions from the rest of the molecule. A detailed discussion on this subject can be found in ref. [21] from which the experimental results in Fig. 7 and 8 were taken. The three basic scattering functions of a subunit of the E-coli ribosome are shown in Fig. 7 whose experimental verification and meaning was discussed above. 


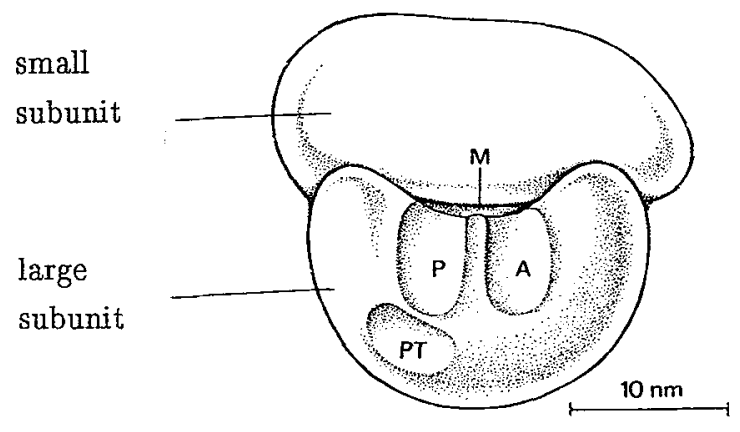

Fig. 5

Model of a ribosome E-coli.

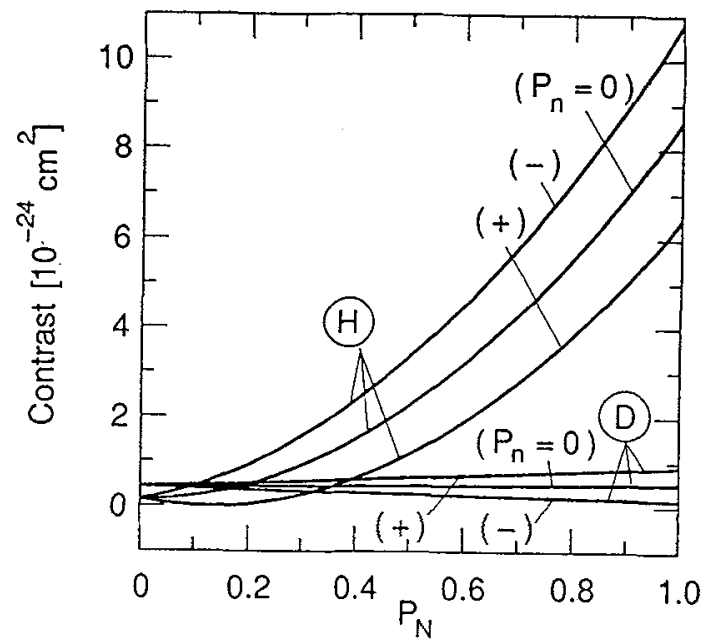

Fig. 6

Contrast of $H$ and $D$ in vacuum as a function of nuclear spin polarization $\left|\underline{\underline{p}}_{N}\right| \cdot+,-$ means neutron spin parallel and antiparallel to the nuclear spin, respectively. 0 means unpolarized 


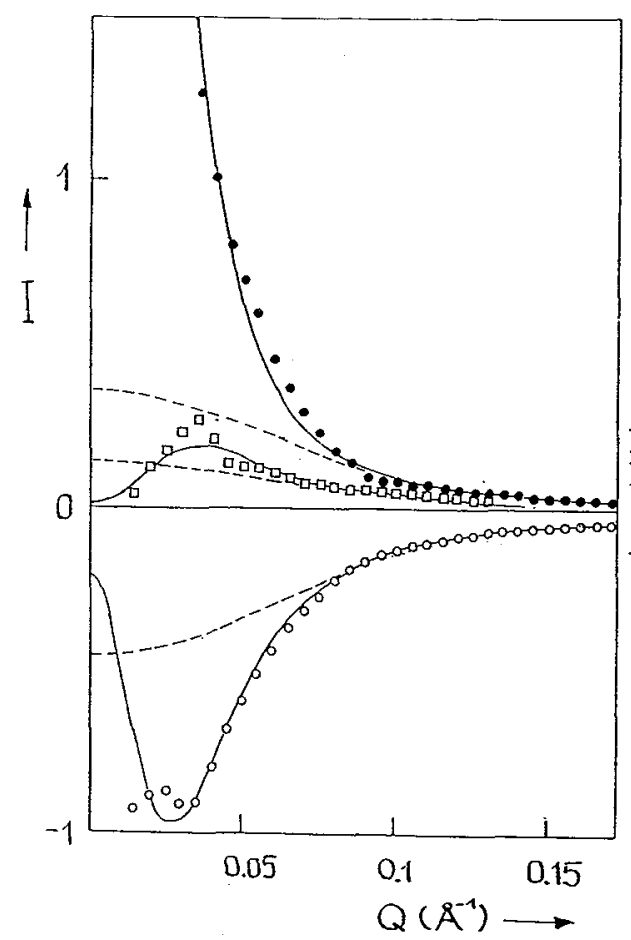

Fig. 7

Basic scattering functions $\left(-\square-|\mathrm{U}|^{2}\right.$, $\left.\left|\mathrm{F}_{\mathrm{A}}\right|^{2},-\mathrm{O}-\operatorname{Re}\left\{\mathrm{UF}_{\mathrm{A}}{ }^{*}\right\}\right)$ of the L3 subunits in the E-coli ribosom shown in Fig. 8 [21].
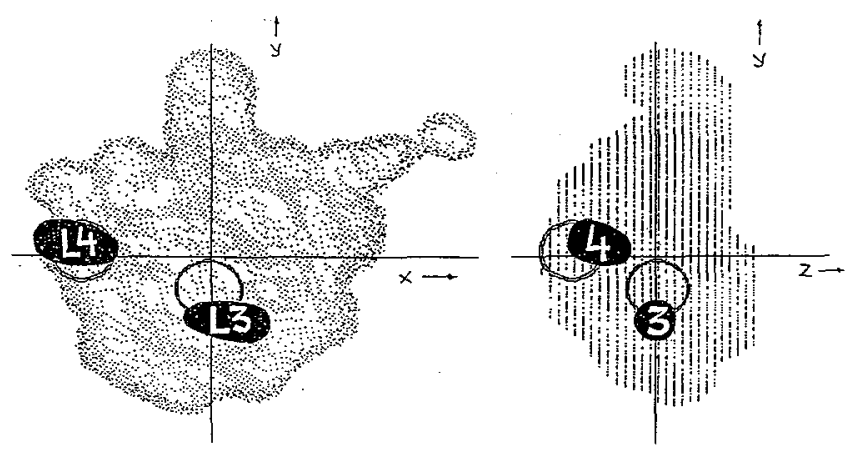

Fig. 8

Positions of L3 and L4 proteins in the large subunit of $\mathrm{E}$-coli ribosome as determined by nuclear contrast variation [21]. 
In total SANS studies on two protein subunits which the authors call L3 and L4 were performed [21]. Therefore three samples with L3, L4, and L3 plus L4 protonated and the rest of the molecule deuterated were prepared. From these studies the knowledge of the architecture of the E-coli ribosome could be developed. The result is compared with earlier TEM studies as marked by the circles in Fig. 8 [21].

\section{Summary}

The contrast variation using polarized neutrons in SANS was discussed. Examples from metal physics and biology using magnetic and nuclear spin polarization have been presented. The main advantages of contrast variation with polarized neutrons and targets are that only one sample is needed and several contrast combinations are available; in certain cases a strong increase of contrast is achieved. At present, only few groups use this technique. On the basis of the advantages discussed here the application of polarized neutrons in SANS may become of importance in many fields of biology and also in metal physics.

\section{Acknowledgement}

I would like to thank Prof. H. Stuhrmann for his permission of presenting the data in Fig. 7 and 8 , and Prof. T. Springer and Stefan Janßen for critical reading the manuscript.

\section{References}

[1] ULLMAIER H., "Irreversible Properties of Typ II Superconductors", Springer-Verlag Berlin 1975.

[2] FORGAN E.M., Mck PAUL D., MOOK H.A., TIMMINS P.A., KELLER H., SUTTON

S., and ABELL J.S., Nature 343 (1990) 735.

[3] KOMURA S., TAKEDA T., SCHWAHN D., and SPRINGER T., Solid State Communications 74 (1990) 965.

[4] TOPERVERG B.P. and WENIGER J., Physica B 174 (1991) 349.

5. ZHUCHENKO N.K., Physics Letters A 161 (1992) 545.

SPRINGER T. and SCHMATZ W., Bull. Soc. fr. Minéral. Crystallogr. 90 (1967) 428.

SCHMATZ W., Rivista Del Nuovo Cimento 5 (1975) 398.

SCHMATZ W., DEDERICHS P.H., and SCHEUER H., Z. Physik 270 (1974) 337.

ANDERS R., GIERHRL M., RÖBER E., STIERSTADT K., and SCHWAHN D., Solid State Communications 51 (1984) 111.

[10] DERIGLAZOV V., OKOROKOV A., RUNOV V., TOPERVERG B., KAMPMANN R., ECKERLEBE H., SCHMIDT W., and LÖBNER W., Physica B 180\&181 (1992) 262.

[11] SCHARPF O., Physica B 174 (1991) 514.

BACON G.E, "Neutron Diffraction", 3rd ed, Clarendon Press, Oxford 1975.

Cambridge University Press 1978.

[14] GAVIN WILLIAMS W., "Polarized Neutrons", Clarendon Press, Oxford 1988.

[15] ABRAGAM A. and GOLDMAN M. "Nuclear magnetism: order and disorder", Clarendon Press, Oxford 1982.

[16] EBEL T., KAMPMANN R., and WAGNER R., Physica B 180\&181 (1992) 357.

[17] SCHWAHN D., ULLMAIER H., SCHELTEN J., and KESTERNICH W., Acta Metall. 31 (1983) 2003.

[18] QIANG L., KESTERNICH W., SCHROEDER H., SCHWAHN D., and ULLMAIER H., Acta Metall. 38 (1990) 2383.

[19] KESTERNICH W., SCHWAHN D., and ULLMAIER H., Scriptametall. 18 (1984) 1011.

[20] KNOP W., HIRAI M., OLAH G., MEERWINCK W., SCHINK H.-J., STUHRMANN H.B., WAGNER R., WENKOW-ESSOUNI M., ZHAO J., SCHÄRPF O., CRICHTON R.R., KRUMPOLC M., NIERHAUS K.H., NIINIKOSKI T.O., and RIJLLART A., Physica. B 174 (1991) 275.

[21] STUHRMANN H., Physica Scripta to be published. 